\title{
MicroRNAs as potential biomarkers of smoking-related diseases
}

MicroRNAs (miRNAs) comprise a family of small, endogenous, noncoding functional RNA molecules that have emerged as key post-transcriptional regulators of gene expression. They inhibit the translation of proteins from mRNA or promote its degradation. Aberrant miRNA expression has been linked to various human diseases and measurement can differentiate between normal and diseased tissue. Expression is tissue-specific and any changes in miRNA expression within a tissue type can be correlated with disease status. Altered miRNA expression has been reported in the smoking-related diseases cancer, chronic obstructive pulmonary disease and cardiovascular disease. Additionally, miRNAs are thought to have vital roles in inflammatory cell differentiation and regulation. miRNAs might, therefore, be useful biomarkers for early detection of disease-related molecular and genetic changes. In this review, we summarize the available scientific evidence for the potential of miRNAs as biomarkers of smoking-related diseases. Studies should be carried out to identify the miRNAs most relevant to specific diseases.

\section{KEYWORDS: biomarker cancer cardiovascular disease chronic obstructive pulmonary disease inflammation miRNA smoking}

MicroRNAs (miRNAs) comprise a family of small, endogenous, noncoding functional RNA molecules 18-25 nucleotides in length. Since their discovery in 1993, in Caenorhabditis elegans [1], and later detection in Homo sapiens in 2000 [2], research has deciphered increasing information on miRNAs as regulators of gene expression [3]. More than 1000 miRNA genes are thought to exist in the human genome and account for $1-5 \%$ of all predicted human genes [4]. There are currently a total of 1921 mature human miRNAs and 1157 mature mouse miRNAs listed in miRBase (release 18), the central online repository for miRNA sequences and annotation [101].

miRNAs were first identified through their ability to regulate developmental processes, such as developmental timing and cell-fate transitions [5]. They also play crucial parts in the regulation of cellular homoeostasis and responses to various stimuli [6]. These RNAs can bind to and control cellular concentrations of target mRNA, which is critical to the regulation of protein expression [4]. Individual miRNAs can regulate more than one target transcript, and estimates indicate that they might regulate up to $30 \%$ of all protein-coding genes in the human genome $[4,7]$. Expression is tissue-specific, and any changes in miRNA expression within a tissue type can be correlated with disease status [8].

Under a standard nomenclature system, miRNAs are assigned sequential numerical identifiers.
The prefix ' $m i r$ ' is followed by a dash and a number, the latter often indicating order of naming. Abbreviated three or four letter prefixes are used to designate the species, such that identifiers take the form hsa-miR-101 (in Homo sapiens). In the miR Base repository, the mature sequences are designated 'miR' whereas the precursor hairpins are labelled 'mir'. The gene names are intended to convey limited information about functional relationships between mature miRNAs. For example, hsa-miR-101 in human and mmu$m i R-101$ in mouse are orthologous. Paralogous sequences whose mature miRNAs differ at only one or two positions are given lettered suffixes, for example, mmu-miR-10a and mmu-miR-10b. Distinct hairpin loci that give rise to identical mature miRNAs have numbered suffixes (e.g., dme-mir-281-1 and dme-mir-281-2 in Drosophila melanogaster) [9].

miRNAs are involved in virtually every cellular process, and aberrant expression is associated with cellular dysfunction and disease [10]. Cigarette smoking causes various molecular and genetic changes in the respiratory tract and is a high risk factor for diseases such as lung cancer [11], chronic obstructive pulmonary disease (COPD) [6] and atherosclerosis [12]. In this review, we discuss miRNAs as potential biomarkers for these major smoking-related diseases and their roles in the innate inflammatory response, in general and in the context of smoking.

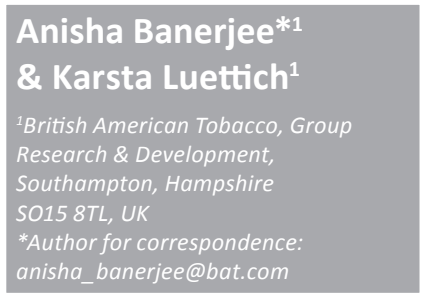

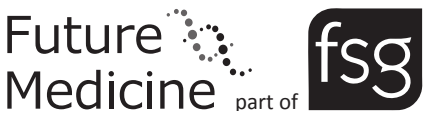




\section{Cancer}

Carcinogenesis is a multistep process characterized by the following distinct stages: initiation, promotion, progression, malignant conversion and formation of a fully developed tumor [13]. Genetic mechanisms have been identified in the deregulation of miRNA that potentially lead to the development of cancer [14-18]. Chromosomal abnormalities that are associated with the deletion, amplification or translocation of miRNAs are typically involved. A significant association between the chromosomal location of miRNAs and those of mouse susceptibility loci that influence the development of solid tumors was shown by Sevignani and colleagues [19].

Additionally, approximately $50 \%$ of all annotated human miRNA genes are located at fragile sites of chromosomes that are prone to breakage and rearrangement in cancer cells [20]. For instance, downregulation of two miRNAs, $m i R-15 a$ and $m i R-16-1$, located at 13q13.4, has been reported in $70 \%$ of patients with chronic lymphocytic leukemia [21].

The processing and stability of miRNAs are important factors that determine their expression levels. In several cancers, expression levels of the ribonucleases Drosha and Dicer1, which are involved in the generation of miRNAs (FIGURE 1), are altered [20]. For example, Melo and Esteller reported that Drosha upregulation is seen in more than half of the samples from cervical squamous cell carcinoma [20]. Expression of some miRNAs in cervical squamous cell carcinoma is, however, reduced even when Drosha is overexpressed. This finding indicates that individual miRNAs respond differently to changes in expression of various regulatory components. The interaction of Drosha with an oncogenic fusion protein also influenced miRNA expression patterns [20]. Additionally, alterations in miRNA expression alone can cause cells to become neoplastic. For instance, overexpression of $m i R-155$ can cause B-cell lymphomas [22], and under-expression of mir-15a in the miR-15/16 cluster in a knockout mouse led to development of chronic lymphocytic leukemia [23].

Global expression of miRNA in tumors is mostly reduced compared with that in normal tissues [24-27]. Yanaihara and colleagues studied more than 100 matched pairs of primary malignant and normal adjacent lung tissue samples and found that the expression in tumor samples differed significantly for 43 miRNAs: 28 were downregulated and 15 were upregulated [28].

Shen et al. analyzed miRNA profiles of human bronchial epithelial cells malignantly transformed by benzo(a)pyrene-trans-7,8-diol- $\alpha-9,10$-epoxide, an important mutagenic metabolite of benzo(a) pyrene [29]. The expression of $54 \mathrm{miRNAs}$, of which 45 were overexpressed and nine were downregulated, differed from that of miRNAs in control cells. This finding was in contrast to the findings of Yanaihara and colleagues, where most of the miRNAs were downregulated. Of note, the target gene of one downregulated miRNA, $m i R-10 a$, was upregulated three- to nine-fold in the transformed cells [29].

\section{miRNAs as oncogenes \& tumor suppressors}

The effects of altered miRNA expression can precede tumor formation, which suggests that miRNAs can function as oncogenes or tumor suppressor genes [11]. A 24-week exposure of Fisher 344 rats to tamoxifen, a potent hepatocarcinogen in these animals, led to substantial upregulation of miRNAs, such as the miR-17-92 cluster (comprising seven miRNAs), miR-106a and miR-34. Moreover, corresponding changes were seen in the expression of target proteins implicated in carcinogenesis, such as cell cycle regulators, chromatin modifiers and expression regulators [30]. Overexpression of the miR-17-92 cluster has also been reported in the development of lung cancers, especially aggressive small-cell cancers [31].

Amplification or deregulation of transcription factors, or demethylation of $\mathrm{CpG}$ islands (regions of DNA containing high numbers of CpG sites) in the promoter region of a miRNA gene can result in overexpression of the miRNA in human cancer $[14,15,17,18,20,32]$. In turn, overexpressed miRNAs can function as oncogenes by downregulating tumor-suppressor genes that control cell differentiation and apoptosis [32]. Some oncogenic miRNAs seem to exert their functions through the blockade of cell cycle inhibitors, such as members of the inhibitor of kinase 4 or cyclin-dependent-kinase-interacting protein/kinase interacting protein families. Most miRNAs with tumor-suppressing abilities exert their functions in several malignancies and through multiple targets [20].

The most abundant cancer-related miRNA with oncogenic properties appears to be $m i R-21$, which is upregulated in more than 15 different cancers, including aggressive lymphoma, pancreatic and lung cancers [6,13]. This miRNA regulates the anti-apoptotic activity of the tumor suppressor genes PTEN and PDCD4 $[33,34]$. Inactivation of $m i R-21$ in breast cancer and breast cancer cell lines increases cell death rates by reactivation of caspases [13]. 


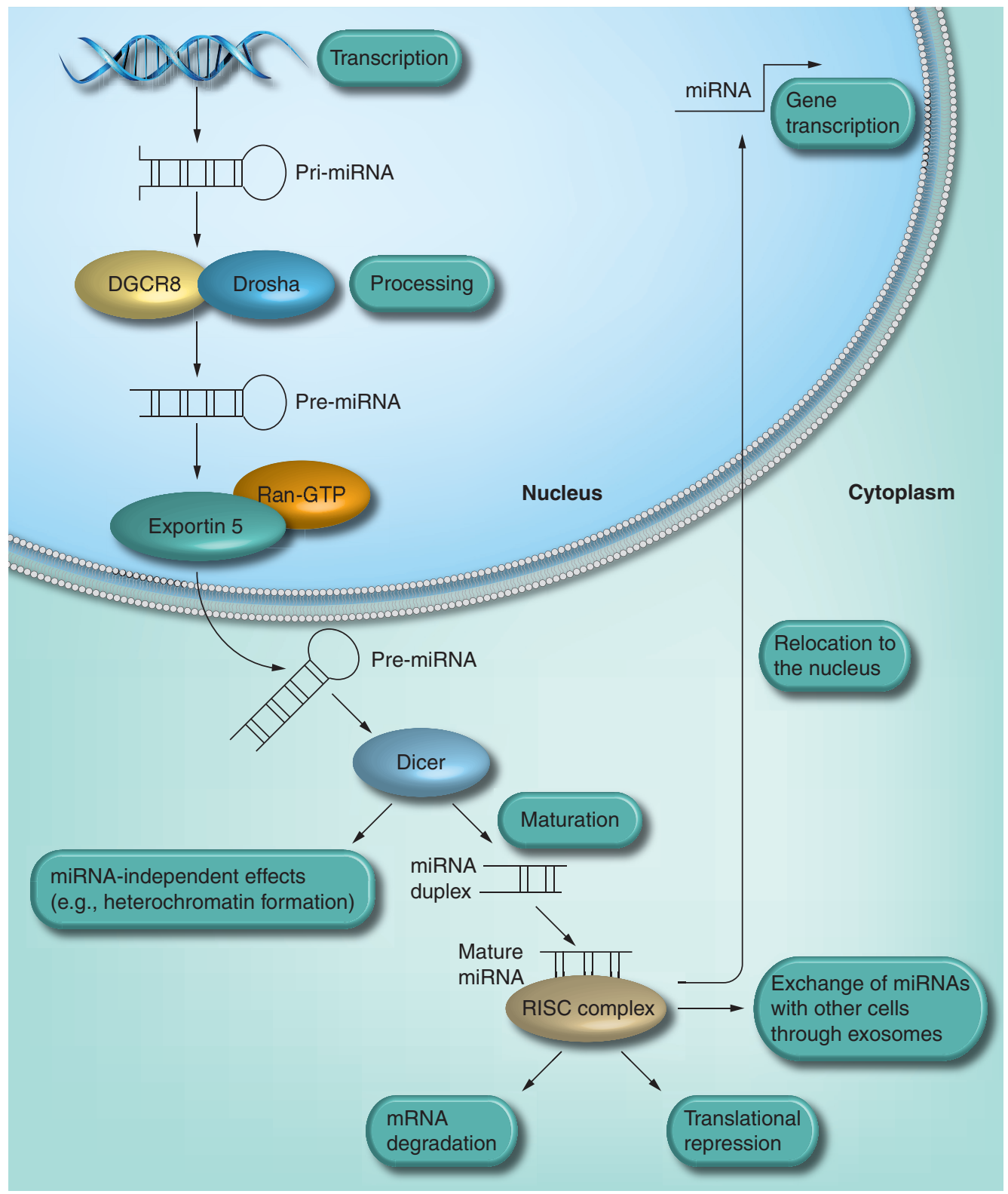

Figure 1. MicroRNA biogenesis. The maturation of miRNAs is mediated by the two RNase III endonucleases, Dicer and Drosha. In the first step, the microprocessor complex composed of Drosha and DGCR8 mediates the nuclear processing of the pri-miRNAs into stem-loop precursors of approximately 60-70 nucleotides (pre-miRNA). The nuclear export of the precursors is subsequently mediated by exportin 5 in a Ran-GTP-dependent manner. In the second step, the pre-miRNA is cleaved in the cytoplasm by Dicer into the mature miRNA, which is approximately 22 nucleotides in length and incorporates as ssRNA into the RISC complex. This complex directs the miRNA to the target mRNA, which leads either to translational repression or degradation of the target mRNA. miRNAs are also localized to the nucleus, where they may regulate transcription or splicing of transcripts. In addition, exosomes mediate the transfer of miRNAs. miRNA: MicroRNA; Pri-miRNA: Primary miRNA; RISC: RNA-induced silencing complex. Reproduced with permission from [53].

Upregulation of the miR-17-92 cluster correlates with increased cell proliferation and decreased apoptosis [35]. Additionally, in irradiated mice that received hematopoietic stem cells expressing the miR-17-19b-1 cluster (the vertebrate-specific segment of the $m i R-17-92$ cluster) and the $M y c$ oncogene, malignant lymphoma developed more rapidly than in those that received stem cells expressing $M y c$ alone. The lymphomas from the former group of mice were also characterized by increased cell proliferation and decreased apoptosis levels [7].

Another miRNA that is overexpressed in multiple cancers (e.g., chronic lymphocytic leukemia, 
B-cell lymphoma, Hodgkin's lymphoma and breast cancer) is $m i R-155$ [35]. Furthermore, when Iorio and coworkers analysed tissue samples from 76 breast cancers and ten normal breasts, miR-155 was among the miRNAs that showed high and consistent upregulation, which supports an oncogenic role [25].

Of the miRNAs that yield tumor-suppressive effects, the let-7 family was one of the first identified with a role in human lung cancers [6]. Certain let-7 homologs are substantially downregulated in human lung cancer, and low levels of let-7 correlate with poor prognosis [28,36]. Moreover, transient expression of let-7 miRNAs in lung adenocarcinoma cell lines inhibits colony formation [36]. The let-7 family of miRNAs also interacts with the Ras protein family, which has oncogenic activity, with reciprocal expression having been seen in lung tumor samples - that is, let-7 overexpression has been associated with decreased Ras protein concentrations in human cancer cell lines [7]. This regulation is achieved through direct interaction between let-7 and the 3' untranslated region of RAS mRNA [7].

The miRNA miR-30e is thought to have tumor-suppressing properties. When miR-30e was overexpressed in HeLa and $293 \mathrm{~T}$ cells, expression of the E2-conjugating enzyme Ubc9 was suppressed, which suggests that the enzyme is a direct target of this miRNA [37]. Ubc9 is essential for post-translational SUMOylation, which contributes to regulation of protein stability, protein-protein interactions, transcriptional activity and subcellular localization [38]. In addition, Ubc9 targets cell cycle proteins, oncogenes and tumor suppressors. The ability of $m i R-30 e$ to negatively regulate $\mathrm{Ubc} 9$ suggests indirect control of cell proliferation and survival [37].

The effect of miRNAs on gene expression in the p53 network is also of importance. The p53 tumor-suppressor co-ordinates various cellular responses to cancer-initiating stressors, including changes in expression of miRNAs. Chang and coworkers treated cells with DNA-damaging agents and noted that $\mathrm{p} 53$ upregulated various miRNAs, of which miR-34a was affected the most [14]. Raised expression of $m i R-34 a$ resulted in increased apoptosis and altered expression of genes related to cell cycle progression, apoptosis, DNA repair and angiogenesis.

Other cell cycle factors in human cancers are subject to regulation by miRNAs. The retinoblastoma protein pathway is altered in almost every human cancer. Retinoblastoma protein is abnormally downregulated by the overexpression of miR-106a in multiple cancers [39]. Moreover, the positive regulators of the cell cycle - cyclins and cyclin-dependent kinases (CDKs) - are targeted by miRNAs. For example, cyclin D1 and CDK6 protein levels were downregulated by induction of G1 cell cycle arrest by $m i R-34 a$ in the A549 lung carcinoma cell line, and cell cycle arrest is promoted by $m i R-34 a$ when CDK 4 and cyclin E protein levels fall [16]. CDK6 is also targeted by $m i R-124$ and $m i R-137$, which are both silenced by $\mathrm{CpG}$ island hypermethylation in tumor cells [20].

\section{miRNAs \& metastasis}

Approximately $90 \%$ of cancer deaths are caused by metastasis, which arises owing to morphological changes, such as cellular disengagement, motility from the local microenvironment, degradation of the extracellular matrix and cellular movement [13]. Co-ordination of some of these pathways by miRNAs plays an important part in tumor invasion and metastasis [32].

Epithelial-mesenchymal transition is one such critical step in tumor invasion and metastasis regulated by miRNAs. The miR-200 family is thought to have a major role in controlling the epithelial phenotype by prevention of $Z E B 1$ and $Z E B 2$ expression. These genes regulate transcriptional repression of E-cadherin, a transmembrane protein involved in cell adhesion [40]. In response to treatment with TGF- $\beta$, a potent inducer of epithelial-mesenchymal transition, $Z E B 1$ and $Z E B 2$ are activated and repress the expression of their own suppressor, miR-200, thereby promoting epithelial-mesenchymal transition, invasion and metastasis [40].

Kong and coworkers showed that breast cancer tissues contain an elevated level of miR-155 [41]. Knockout of this miRNA inhibits TGF- $\beta$ induced epithelial-mesenchymal transition, tight-junction dissolution and cell migration and invasion. Thus, $m i R-155$ is thought to play an important role in these processes [32].

\section{COPD}

COPD is predominantly induced by chronic exposure to irritants, including cigarette smoke. This disease is the fourth-leading cause of death in the USA [42] and has been predicted to increase to third place by 2020 [43]. Hallmarks of COPD include progressive obstruction of airflow, chronic inflammation in the lungs, shortness of breath, chronic cough and phlegm production [44]. Additionally, repair of injured lung tissue is hindered [6]. Airway and systemic inflammation have been attributed to the pathogenesis of COPD [44]. Smoking cessation at an 
early stage of the disease can slow the loss of lung function, but an abnormal inflammatory response persists [45].

Sato et al. reported a potential role for $m i R-146 a$ in the abnormal inflammatory response observed in COPD [45]. In primary lung fibroblasts isolated from 14 smokers with mild-to-severe disease, expression of $m i R-146 a$ was low following stimulation with IL-1 $\beta$ and TNF- $\alpha$, compared with that in fibroblasts from 16 smokers without COPD. As a result COX2 mRNA was degraded and production of prostaglandin E2, an inflammatory mediator whose expression is increased in the lungs of COPD patients, was increased.

In a study of current smokers with COPD and individuals who had never smoked and had no airflow limitation, eight miRNAs were differentially expressed in sputum supernatant [46]. Of these, two - let- $7 c$ and $m i R-125 b$ - were confirmed in a validation cohort to differ significantly between groups. Reduced expression of let- $7 c$ inversely correlated with sputum concentrations of TNF receptor II (TNFR-II), a primary target of this miRNA, which suggests that $l e t-7 c$ inhibits translation of its target genes. Of particular note is that let-7c was expressed in bronchial epithelial cells and macrophages, which are involved in the pathogenesis of COPD and express TNFR-II. Recently, Akbas and colleagues identified miRNA in serum of COPD patients and found the expression of $m i R-7$ to be significantly upregulated, while $m i R-20 a$, $m i R-28-3 p, m i R-34 c-5 p$ and $m i R-100$ expression was downregulated compared with healthy controls. Although a small study, these preliminary data suggest that the identified miRNA might serve as potential biomarkers of disease [47].

COPD generally affects older individuals, owing to the duration of smoking. Whether age is a risk factor for the disease is unclear. In murine models of lung aging in healthy mice, however, expression of miRNAs seemed to remain stable throughout adulthood [48]. Thus, miRNAs might be quiescent while lung homeostasis is maintained after development, but may become perturbed in disease states involving cell differentiation and inflammation [6].

\section{Cardiovascular disease}

miRNAs are of crucial importance for heart development and function [49]. Various cardiovascular diseases (CVDs) have been associated with changes in miRNA expression (Figure 2) [50]. Aberrant expression of miRNAs was seen in the vascular wall after balloon injury during angioplasty in rats [51], and further roles for miRNAs have been seen in other disorders of CVD, such as atherosclerosis, and in disease processes, such as angiogenesis.

\section{Atherosclerosis}

Atherosclerosis is one of the leading causes of mortality in the developed world [52]. In this chronic inflammatory disease, injury to the vessel wall leads to endothelial permeability, upregulation of leukocyte and endothelial adhesion molecules, leukocyte recruitment and migration into the artery wall, formation of fatty streaks, platelet aggregation, smooth muscle cell migration and proliferation within the intermediate lesion and the gradual formation of the atherosclerotic plaque. Complications are plaque rupture and thrombosis [53].

Endothelial expression of VCAM-1, which mediates leukocyte adherence to the vascular endothelium, is inhibited by miR-126 [54]. Conversely, reduction in miR-126 expression is associated with increased TNF- $\alpha$-stimulated expression of VCAM-1 and, thereby, increased adherence of leukocytes to endothelial cells. These findings indicate a role in vascular inflammation. Another miRNA, miR-21, regulates neointimal lesion formation. Ji et al. found that the number of differentially expressed miRNAs changed during lesion formation - of 140 arterial miRNAs assessed, 113 were differentially expressed 7 days after balloon injury in rats, 110 at 14 days and 102 at 28 days; and miR-21 was one of the most upregulated miRNAs [55]. Inhibition of $m i R-21$ expression decreased proliferation and increased apoptosis of vascular smooth muscle cells in vitro and in injured rat carotid arteries in vivo [55].

The angiotensin II type 1 receptor controls blood pressure and volume in the cardiovascular system. Angiotensin II stimulates the receptorassociated and protein kinase C-activating Gq protein in vascular smooth muscle cells, which leads to increased blood pressure. This disorder is a key contributor to the development of CVD. Martin et al. found that miR-155 was expressed in vascular smooth muscle cells, and transitionally repressed angiotensin II type 1 receptors in these cells [56]. In addition, miR-155 is induced in macrophages by cytokines and contributes to physiological expansion of granulocytes and monocytes during inflammation [57].

According to Pasterkamp et al. [58], toll-like receptors (TLRs), especially TLR4, have crucial roles in the development and destabilization of atherosclerotic plaques and seem to be miRNA 


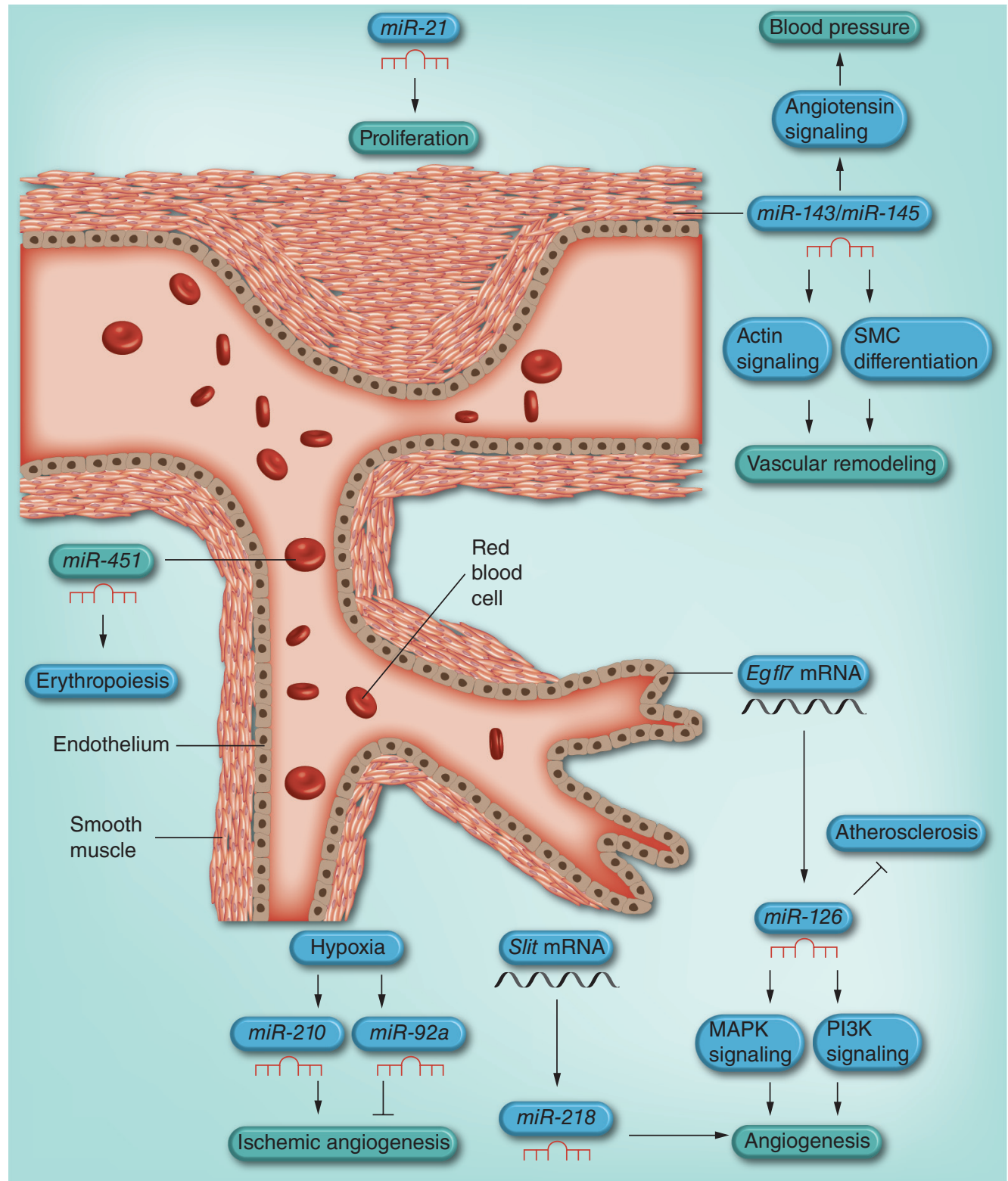

Figure 2. Roles of microRNAs in cardiovascular disease processes. The endothelial and smooth muscle layers, red blood cells and the proliferating SMCs of a neointimal lesion. The expression of select miRNAs is shown, along with their observed functional role. Hypoxia results in the activation of miR-210 and miR-92a, which promote and inhibit angiogenesis, respectively. miR-126, an endothelial cell-enriched miRNA encoded by an intron of the Egfl7 gene, modulates atherosclerosis and angiogenesis by regulating MAPK and PI3K signaling. Angiogenesis is also regulated by miR-218, which is encoded by an intron of the Slit genes. miR-143 and miR-145 are expressed in SMCs and control blood pressure and vascular tone, and contribute to vascular remodeling. miR-21 is induced in SMCs after vascular injury, and promotes proliferation and neointima formation. miR-451 regulates the proliferation and differentiation of erythroid cells.

SMC: Smooth muscle cell.

Reproduced with permission from [96].

targets [52]. Chen et al. reported translational regulation of TLR 4 by the miRNA let-7i in a cell culture model of cholangitis [59]. Furthermore, $m i R-146$ is part of a negative feedback loop in monocytes that is stimulated by lipopolysaccharides (LPSs) and controls TLR and cytokine signaling [60].

\section{Vascular angiogenesis}

Angiogenesis is the growth of new blood vessels from pre-existing vessels, by spontaneous growth or by the splitting of existing vessels. The physiological process involves proliferation, migration and maturation of endothelial cells [53]. Angiogenesis, however, can become pathophysiological, and in 
CVD it is closely related to destabilization and rupture of atherosclerotic plaques [52].

Dicer has a role in miRNA maturation (Figure 1) and has also been shown to have a role in angiogenesis [53]. Genetic deletion of Dicer in embryonic mouse cardiomyocytes results in abnormal blood vessel formation [61] and yolk sac vascularization [62], and silencing in endothelial cells substantially reduced angiogenesis [63]. In addition, knockdown of Dicer in endothelial cells prevented capillary sprouting and tube formation $[53,61]$. Some miRNAs are known to affect angiogenesis through positive and negative regulator roles [52,61]. According to Bonauer et al., human endothelial cells express the $m i R-17-92$ cluster [64]. Overexpression of one of the seven miRNAs in this cluster, miR-92a, blocked angiogenesis in vivo and in vitro. By contrast, in the same study, when $m i R-92 a$ was inhibited, blood vessel growth in ischemic limbs improved and infarct size was reduced after myocardial infarction in mice. Prevention of induced gene silencing can, therefore, improve collateral vessel development [65].

Among highly expressed miRNAs in human umbilical vein endothelial cells, $m i R-221$ and miR-222 exert anti-angiogenic effects. These miRNAs are activated by stem cell factor and are thought to downregulate expression of the proto-oncogene $c$-Kit, which leads to decreased cell survival, migration and endothelial tube formation [66]. Additionally, expression of other miRNAs in these cells changes when miR-221 and $m i R-222$ are depleted, which suggests that miRNAs control the expression of other miRNAs [67]. On the other hand, when miR-221 and $m i R-222$ were overexpressed, expression of endothelial nitric oxide synthase, which generates nitric oxide in blood vessels, was indirectly lowered in endothelial cells after silencing of Dicer by siRNA [68]. Nitric oxide is a key regulator of endothelial cell growth, migration and angiogenesis, and its bioavailability is impaired in patients with atherosclerosis [53].

Genetic deletion of $m i R-126$, which is highly enriched in mouse endothelial cells derived from embryonic stem cells or developing embryos [69], resulted in vascular development defects, such as delayed angiogenic sprouting and widespread hemorrhaging [3]. Angiogenic factors, including VEGF, EGF and bFGF, had no effects on $m i R$-126-deficient endothelial cells [3]. SPRED-1 and the regulatory subunit of PI3K, p $85 \beta$, have been identified as the two direct targets of miR-126 [61,69]. These proteins negatively regulate VEGF signaling via the MAPK and PI3K pathways, respectively, which inhibit angiogenesis. Therefore, miR-126 promotes signaling of this and possibly other growth factors (Figure 3) [69] and contributes to vascular integrity and promotion of vessel growth [61].

Fasanaro et al. reported that blockade of $m i R-210$ inhibits hypoxia-induced vessel formation and is an important factor in endothelial cell migration driven by VEGF [70]. This growth factor is also a target of $m i R-15 b, m i R-16$, $m i R-20 a$ and $m i R-20 b$, which are sharply downregulated after hypoxia. Inhibition of these posttranscriptional regulators led to greatly increased VEGF expression, which suggests that miRNAs contribute to the angiogenic switch in response to hypoxia [52].

\section{Pulmonary hypertension}

Pulmonary hypertension (PH) is characterized by constricted and remodeled pulmonary vasculature leading to increased blood pressure in the lung, and can ultimately result in cardiac failure. Based on the current understanding of $\mathrm{PH}$, the WHO classifies this disease as pulmonary arterial hypertension (PAH), PH associated with left heart disease, $\mathrm{PH}$ associated with lung diseases or hypoxemia, thromboembolic $\mathrm{PH}$ or $\mathrm{PH}$ linked to other diseases [71]. Cigarette smoke exposure can cause PAH in rodents, and $\mathrm{PAH}$ is thought to occur frequently in COPD

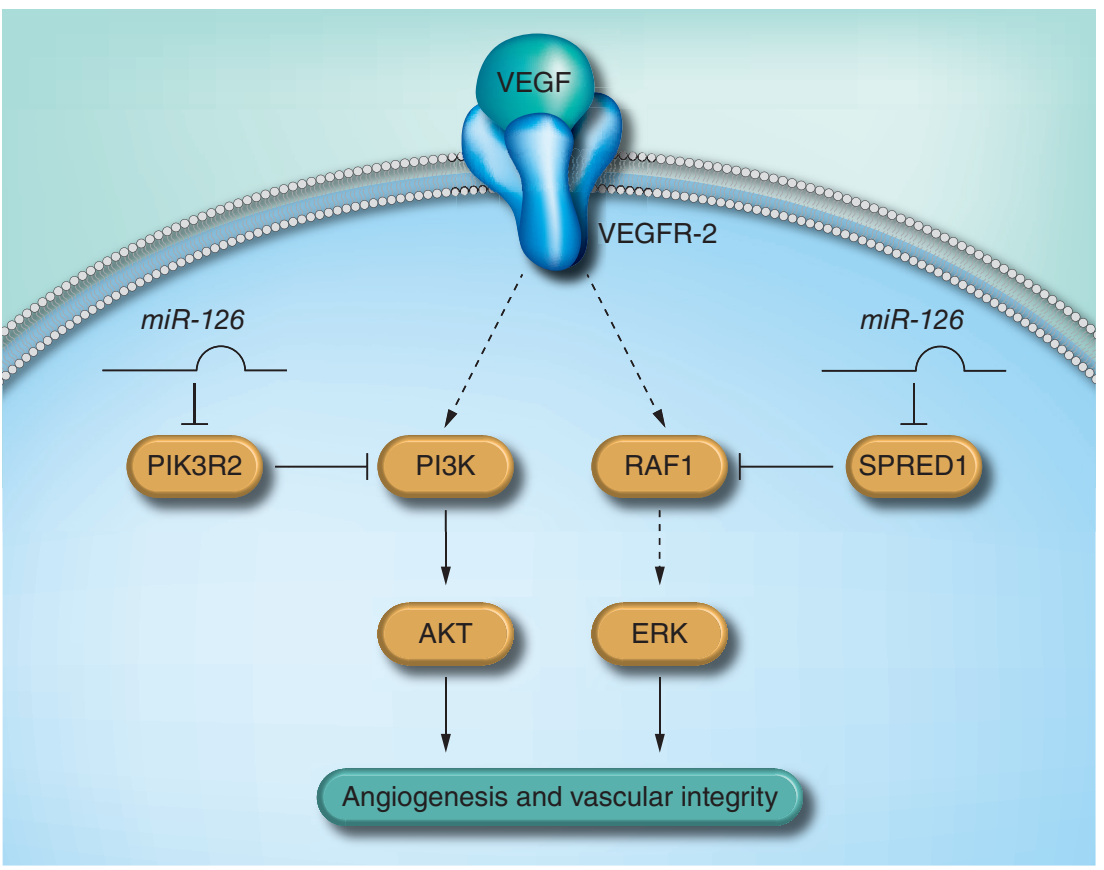

Figure 3. Role of $\boldsymbol{m i R}$-126 in VEGF signaling. SPRED1 and PIK3R2 inhibit signaling of VEGF, and possibly other growth factors, via the MAPK/ERK and PI3K pathways, respectively. Growth factor signaling is, therefore, promoted by miR-126, which leads to increased angiogenesis and vascular integrity.

Reproduced with permission from [97] 
patients [72]. The involvement of miRNAs in the pathogenesis of $\mathrm{PH}$ is unclear. Animal model studies suggest that miRNA expression is deregulated both over time and dependent on etiological aspects. For example, Caruso and coworkers surveyed miRNA expression in total lung extracts from two rat models (chronic hypoxia and monocrotaline model) of PAH [73] A number of miRNAs were found to be differentially expressed in the two disease models. For example, $m i R-21$ was downregulated in the monocrotaline-induced rat PAH model and in lung samples from patients with idiopathic PAH. In addition, $m i R-451$ was upregulated in both rat models, but there were no differences between lung samples from control and PAH human subjects. This initial finding showed that there were disease-related changes in miRNA expression patterns associated with the development of PAH in both animals and humans [73]. Another study showed that $m i R-204$ was also downregulated in the rat monocrotaline model and in the lungs of PAH patients. Moreover, decreased miR-204 levels were found in blood from patients with PAH compared with nonPAH patients, suggesting that this miRNA may serve as a potential biomarker of the disease [74]

\section{Regulation of the inflammatory response}

Inflammation contributes to the development of all the smoking-related diseases discussed in this review [44,53], and resolution is necessary to prevent chronic disease states [44]. A vital role has been suggested for miRNAs in inflammatory cell differentiation and regulation as part of the immune response (Figure 4) [6].

The innate immune response is the first line of host defense and is mediated via myeloid cells, such as macrophages and dendritic cells. The presence of pathogens is detected by pattern recognition receptors that bind conserved molecules within bacteria, fungi and viruses [75]. One such family of recognition receptors is the TLRs, which are of interest in miRNA studies [76]. Taganov et al. screened for upregulated miRNAs in a monocytic cell line treated with the TLR4 ligand LPS [60]. Three miRNAs were upregulated: $m i R-146 a$, miR-155 and miR-132. The researchers speculated that miRNA-146a and $m i R N A-146 b$ act as negative regulators of inflammation through the downregulation of IRAK1 and TRAF6, which are involved in TLR-IL-1 receptor signalling.

LPS has also been shown to induce several other miRNAs, including $m i R-9$ and $m i R-21$
[76]. Perry et al. reported that $m i R-146 a$ and, to a lesser extent, $m i R-146 b$, are central to the negative feedback regulation of IL- $1 \beta$-induced inflammation [77]. This group also demonstrated that the action of these two miRNAs is upon chemokine translation, rather than downregulation of IRAK1 and TRAF6. In another study, direct regulation of TLR4 by let-7i was seen in human biliary epithelial cells, and let-7i expression was reduced by LPS [59]. Two other members of the let-7 family detected in these cells, let-7b and let-7g, are also thought to target TLR4 [59]. These findings suggest that miRNA levels change rapidly to regulate acute biological responses, such as inflammation, and that miRNAs are key regulators of the innate immune response, with particular relevance for TLR signaling.

\section{miRNAs in the context of smoking}

Cigarette smoking causes multiple molecular and genetic changes in the respiratory tract and is a notable risk factor for various diseases, including lung cancer [11], COPD [6] and atherosclerosis [12].

Smoke exposure leads to lung inflammation and an increased number of macrophages in the airway. Few studies have, however, explored lung miRNA responses to cigarette smoke. Taganov et al. reported that expression of $m i R-132$, $m i R-146 a$ and $m i R-155$ were upregulated in macrophage cell lines in response to LPS challenge, a known endotoxin in cigarette smoke [60]. Various alterations in multigene expression are caused in the lungs of mice [48] and rats [78] exposed to cigarette smoke and in airway epithelial cells of humans who smoke [79].

Kalscheuer and colleagues identified miRNA expression changes associated with earlystage neoplastic transformation in the lungs in male F344 rats, chronically treated with 4-(methylnitrosamino)-1-(3-pyridyl)-1-butanone (NNK), a carcinogen present in tobacco products, for up to 20 weeks [80]. Several miRNAs, such as miR-101, miR-126, miR-199 and $m i R-34$ were downregulated after treatment. The authors suggested that these miRNAs could represent early molecular changes associated with pulmonary tumorigenesis. Furthermore, cytochrome P450 2A3, a critical enzyme in rat lungs that catalyzes the metabolism of NNK, which renders it carcinogenic, was identified as a potential target of $m i R-126$. Thus, the carcinogenic effects of NNK could be increased owing to downregulation of $m i R-126$ [80].

Izzotti et al. analyzed the expression of 484 miRNAs in the lungs of rats exposed 
to environmental tobacco smoke (ETS) for 28 days [78]. Of these miRNAs, 126 were downregulated by at least twofold and 24 by more than threefold. The most affected miRNAs had regulatory roles in stress response, apoptosis, proliferation, angiogenesis and gene expression. The rodent miRNAs with deregulated expression showed substantial overlap with their human homologs that are deregulated in smokers, most of which are transcribed from genes located in fragile sites deleted in lung cancer [20]. The authors concluded that these changes indicate early events after exposure to cigarette smoke [78].

The same group examined miRNA expression in mouse lungs that had been exposed to ETS or were untreated. Fifteen miRNAs were downregulated as a result of smoke exposure. Finally, to evaluate the early effects of ETS and of the chemopreventive agents budesonide (BUD) and phenethylisothiocyanate (PEITC), neonatal mice were exposed to smoke immediately after birth and for 2 weeks after weaning, and thereafter either BUD or PEITC were fed to the mice in their diet [81]. Of 576 miRNAs assessed, expression was downregulated by at least twofold in 43 and 90 miRNAs in the lung and liver, respectively, and upregulated by at least twofold in 29 and 76, respectively, in mice exposed to smoke. Some protective effects were seen with BUD and PEITC treatment in the lung, and more so in the liver, mainly from miRNA downregulation.

miRNA alterations were evaluated at a later stage, when lung tumors were detectable in mice exposed to mainstream cigarette smoke (MCS) during the first four months of life, followed by recovery in filtered air for an additional three months [79]. The analysis of 576 mouse miRNAs showed that exposure to MCS extensively dysregulated their expression, and that miRNA patterns were specific for certain histopathologies, in particular pneumonia, adenoma or bronchoalveolar carcinoma. Treatment with the chemopreventive agents PEITC and $N$-acetyl-L-cysteine was shown to attenuate this trend efficiently, especially in noncancer tissue. The alterations of a few miRNAs were expected to trigger multiple mechanisms involved in carcinogenesis, such as oncogene activation, inhibition of oncosuppressor genes, recruitment of undifferentiated stem cells, inflammation, inhibition of gap junctional intercellular communications, angiogenesis, invasiveness and metastasis, suggesting that exposure to MCS is able to dysregulate a

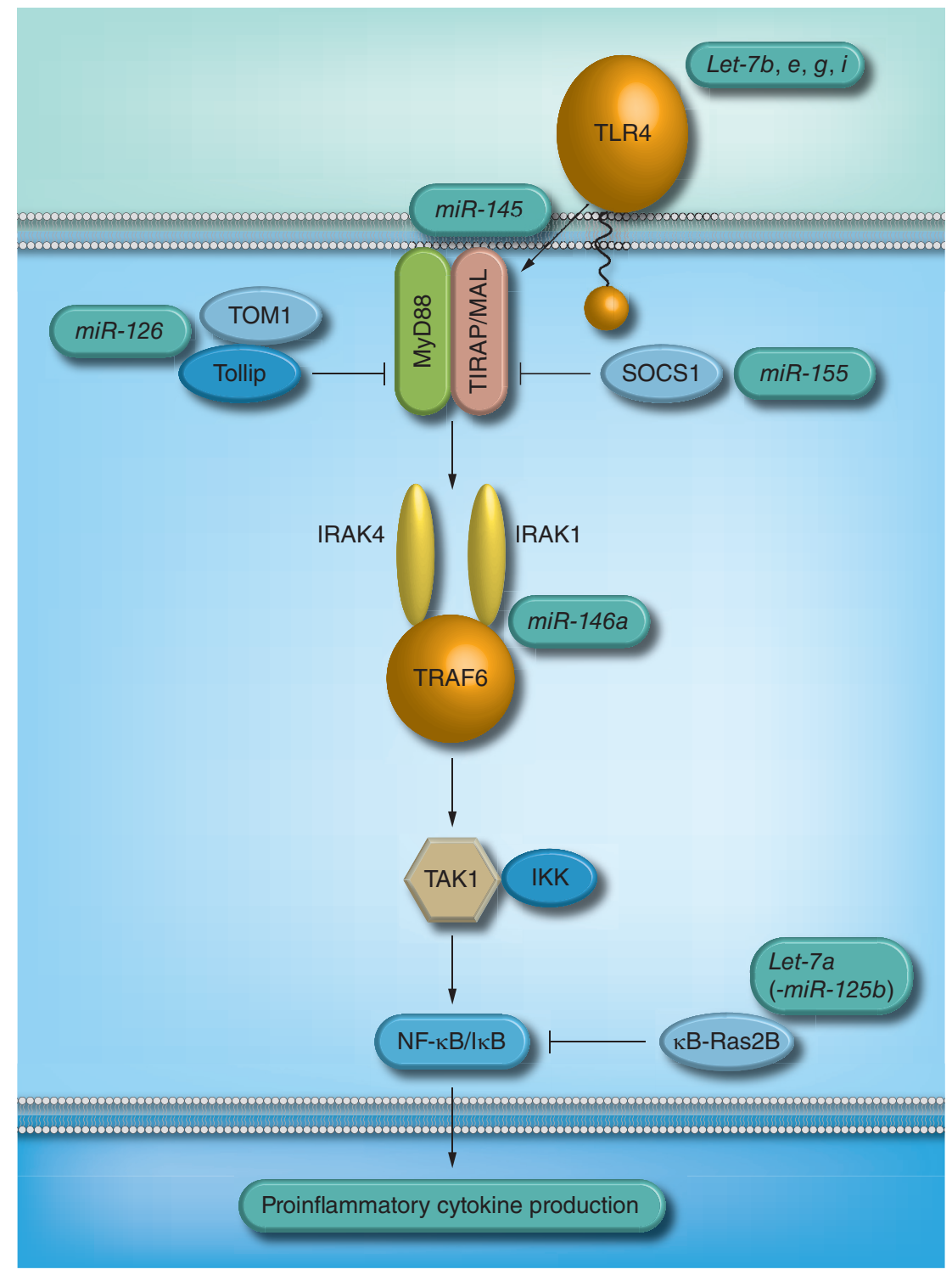

Figure 4. Involvement of microRNAs in innate inflammatory response. TLR4 signals via MyD88 and TIRAP/MAL to IRAK1 and 4. The TOM1/Tollip complex and SOCS1 are negative regulators of this pathway. The signal is transduced via TRAF6, TAK1 and IKK, which leads to activation of NF- $\kappa B$ via dissociation of $I \kappa B$. $\kappa B-$ Ras $2 B$ is an IKB inhibitor. MicroRNAs, shown in boxes, target various components of TLR4 signaling.

Reproduced with permission from [44].

variety of miRNAs throughout all stages of the pulmonary carcinogenesis process [82].

The timeline of cigarette smoke-induced carcinogenesis was another subject of investigation by Izzotti and colleagues [83]. Neonatal mice were exposed to air and three different doses of smoke: 119, 292 and $438 \mathrm{mg} / \mathrm{m}^{3}$ total particulate matter for 4 weeks. Bulky DNA adduct and 8-oxo-7,8-dihydro-2'-deoxyguanosine levels significantly increased in the lungs of animals exposed to low and medium doses of cigarette smoke. However, miRNA expression was not altered. Only at the highest dose 
did the miRNA expression change significantly. This apparent dose-response behavior indicated that a threshold dose of cigarette smoke is required to alter miRNA expression and induce miRNA-mediated carcinogenesis. Interestingly, when cigarette smoke was withdrawn (high-dose exposure group), miRNA levels were seen to increase as soon as 1 week after cessation. A total of 4 weeks of smoke exposure, even at the highest dose, was insufficient to induce irreversible changes in miRNA expression [83].

The reversibility of cigarette smoke-induced miRNA changes in the lung could be a manifestation of the adaptive response mechanisms of lung cells [84]. Short exposure to cigarette smoke might cause downregulation of many miRNAs such as $m i R-122 a, m i R-431$ and $m i R-99 b$ to activate adaptive and protective functions aimed at defending the respiratory tract from the adverse effects of smoke [84].

In another study, Schembri et al. examined whole-genome miRNA and mRNA expression in bronchial airway epithelium from 20 volunteers, of whom ten were current smokers and ten were never-smokers [85]. Expression of 28 miRNAs differed between groups, mostly by downregulation in smokers. Expression of $m i R-218$ was altered the most, showing fourfold downregulation. By contrast, expression of putative targets of these miRNAs was generally increased in smokers. The differentially expressed mRNAs were associated with regulation of oxidative stress, xenobiotic metabolism, inflammation, oncogenesis and tumor suppression.

Christenson and coworkers explored the effects of smoking on miRNA expression in extrathoracic (buccal and nasal) airway epithelium in five healthy current smokers, five former smokers and five never-smokers [86]. A substantial number of differentially expressed miRNAs were identified in buccal and nasal epithelium of all smokers, but for most of these expression levels were reverting in former smokers towards those seen in never-smokers.

Smoking cessation has positive effects on cytomorphology [87]. Quitters have significantly lower mean levels of columnar cells, mucus, mucous spirals and metaplasia in their upper airways than current smokers. The understanding of cessation biology in bronchial epithelial cells is growing steadily, but little is known about the effects on miRNAs. Izzotti and coworkers suggest that let-7 downregulation could be reversible in the early stages of smoking-related disease, and would only become irreversible in cancer-committed cells [48]. Another study by Moriyama et al. reported that smoking cessation and age interacted and affected miRNA profiles [88]. In bronchiolar epithelium following cigarette smoke exposure for 10 days, they found 45 downregulated miRNAs in young mice (aged 9 weeks) and 44 in aged mice (aged 69 weeks). After 20 days, four miRNAs, miR-10a, miR-205, miR-340-3p and $m i R-322$, remained downregulated in aged mice, whereas all reverted to baseline levels in young mice.

Besides cigarette smoke exposure, studies have been conducted to examine the miRNA response pattern to other types of inhaled agents, such as particulate matter from combustion exhaust or aerosolized dusts [89]. Human bronchial epithelial cells grown at an air-liquid interface were exposed to diesel exhaust particles by Jardim and coworkers and assessed by miRNA profiling with arrays [90]. The authors found that of the 197 miRNAs that exhibited changes greater than 1.5-fold, 130 were upregulated and only half that number were downregulated. This finding was contrary to the effect of smoking in human airways, which is largely characterized by global miRNA downregulation [85]. Thus, although some miRNAs were induced or repressed, an equal number behaved disparately, which suggests that the epithelial response is exposure-specific [89].

\section{Potential of miRNAs as disease biomarkers}

A biomarker is a characteristic that can be objectively measured and evaluated as an indicator of normal conditions and disease processes or of pharmacological responses [91]. The high stability of miRNAs in tissue and blood, their resistance to RNA degradation and their easy, reproducible detection makes them suitable biomarker candidates [20]. Evidence also indicates that unique patterns of altered miRNA expression provide valuable information in cancer, such as tumor origin, stage and other pathological factors [20] and could, therefore, serve as molecular biomarkers for diagnosis, prognosis and prediction of therapeutic responses [92].

Mascaux et al. showed that early miRNA changes related to bronchial carcinogenesis could be assessed in biopsy samples [93]. Specimens were obtained by autofluorescence bronchoscopy from smokers and nonsmoker controls. Differential expression for a total 69 miRNAs was seen across the following 
stages: histologically normal and normofluorescent or hypofluorescent, hyperplasia, metaplasia, mild, moderate and severe dysplasia, carcinoma in situ and invasive squamous cell carcinoma. Downregulation of $m i R-32, m i R-34 c$ and $m i R-15 a$ increased progressively from normal to squamous cell carcinoma. Other miRNAs, such as $m i R-142-3 p$ or $m i R-9$, were downregulated then upregulated in successive stages, or had altered expression at one stage of carcinogenesis, such as miR-199a or miR-139. Generally, however, miRNA expression first decreased early and then increased in later stages. Additionally, miRNA expression was closely associated with histological classification of low-grade and highgrade preneoplastic lesions and between in situ, and invasive carcinoma [88].

Studies have also shown that miRNAs can be assessed in serum or plasma. Tumor cells secrete cell membrane microvesicles called exosomes into the extracellular space, which can eventually be detected in the blood. These exosomes have been shown to contain miRNAs [94]. Weber and coworkers examined the presence of miRNAs in 12 human body fluids, including plasma, saliva and urine, and found miRNAs in all fluids tested [95]. The total miRNA composition differed in different fluid types, but $m i R-509-5 p$, $m i R-515-3 p$ and $m i R-335$ were among the most abundant miRNAs across these body fluids. Plasma had the highest number of unique miRNA species, followed by saliva. Additionally, miRNA patterns in urine differed by pathophysiological condition. This finding demonstrates the potential for using expression of specific miRNAs in body fluids as informative biomarkers for detecting and monitoring different pathophysiological conditions [95]. The presence of miRNAs in different body fluids also enables easy detection by noninvasive methods.

\section{Conclusion \& future perspective}

Further research is required to identify the miRNAs most relevant to specific diseases and to develop robust isolation and detection methods. So far, the potential of miRNAs as biomarkers has been shown most clearly for cancer. Given the growing evidence in COPD and CVD, however, it seems likely that miRNAs will also be suitable as biomarkers for these diseases.

\section{Financial \& competing interests disclosure}

A Banerjee is an employee of British American Tobacco and $K$ Luettich is a former employee of British American Tobacco. The authors have no other relevant affiliations or financial involvement with any organization or entity with a financial interest in or financial conflict with the subject matter or materials discussed in the manuscript apart from those disclosed.

No writing assistance was utilized in the production of this manuscript.

\section{Executive summary}

\section{Background}

- MicroRNAs (miRNA) are a family of small, endogenous, noncoding functional RNA molecules that serve as key post-transcriptional regulators of gene expression.

miRNA \& smoking-related diseases (cancer, chronic obstructive pulmonary disease \& cardiovascular disease)

- Aberrant miRNA expression has been linked to smoking-related diseases such as cancer, cardiovascular disease and chronic obstructive pulmonary disease.

- miRNAs regulate developmental processes, co-ordinate intricate gene expression pathways, possess the ability to function as oncogenes or tumor suppressor genes, and play important roles in tumor invasion and metastasis.

\section{Regulation of the inflammatory response}

- miRNAs play a vital role in inflammatory cell differentiation and serve as key regulators of immune response.

\section{miRNAs in the context of smoking}

- miRNA expression changes can be associated with early-stage neoplastic transformation.

- Mainstream cigarette smoke dysregulates a variety of miRNAs throughout all stages of pulmonary carcinogenesis.

- Persistence of alterations in lung miRNA expression are directly proportional to the length of exposure to cigarette smoke.

- Cigarette smoke causes irreversible miRNA expression changes only when exposure occurs for a sufficient period of time at high doses surpassing a certain threshold.

\section{Potential of miRNA as disease biomarkers}

- Differential expression of miRNA is observed in diseased and normal tissues.

- Global expression profiling of miRNA in cells and tissues exposed to tobacco smoke confirmed their involvement in smoking-related disease processes and diseases.

Noninvasive sampling is possible from blood, saliva and urine samples. 


\section{References}

Papers of special note have been highlighted as:

- of interest

" of considerable interest

1 Lee RC, Feinbaum RL, Ambros V. The $C$. elegans heterochronic gene lin-4 encodes small RNAs with antisense complementarity to lin-14. Cell 75(5), 843-854 (1993).

2 Pasquinelli AE, Reinhart BJ, Slack F et al. Conservation of the sequence and temporal expression of let-7 heterochronic regulatory RNA. Nature 408(6808), 86-89 (2000).

3 Wang Z, Luo X, Lu Y, Yang B. miRNAs at the heart of the matter. J. Mol. Med. 86(7), 771-783 (2008).

4 George GP, Mittal RD. MicroRNAs: potential biomarkers in cancer. Ind. J. Clin. Biochem. 25, 4-14 (2010).

5 Kloosterman WP, Plasterk RH. The diverse functions of microRNAs in animal development and disease. Dev. Cell 11(4), 441-450 (2006).

6 Nana-Sinkam SP, Karsies T, Riscili B, Ezzie M, Piper M. Lung microRNA: from development to disease. Expert Rev. Respir. Med. 3(4), 373-385 (2009).

7 Stahlhut Espinosa CE, Slack FJ. The role of microRNAs in cancer. Yale J. Biol. Med. 79(3-4), 131-140 (2006).

8 Bartels CL, Tsongalis GJ. MicroRNAs: novel biomarkers for human cancer. Clin. Chem. 55(4), 623-631 (2009).

9 Griffiths-Jones S, Grocock RJ, van DS, Bateman A, Enright AJ. miRBase: microRNA sequences, targets and gene nomenclature. Nucleic Acids Res. 34(Database issue), D140-D144 (2006).

10 Lema C, Cunningham MJ. MicroRNAs and their implications in toxicological research. Toxicol. Lett. 198(2), 100-105 (2010).

11 Chen T. The role of MicroRNA in chemical carcinogenesis. J. Environ. Sci. Health C. Environ. Carcinog. Ecotoxicol. Rev. 28(2), 89-124 (2010).

12 Howard G, Wagenknecht LE, Burke GL et al. Cigarette smoking and progression of atherosclerosis: the Atherosclerosis Risk in Communities (ARIC) Study. JAMA 279(2), 119-124 (1998).

13 Koturbash I, Zemp FJ, Pogribny I, Kovalchuk O. Small molecules with big effects: the role of the microRNAome in cancer and carcinogenesis. Mutat. Res. 722(2), 94-105 (2011).

14 Chang TC, Wentzel EA, Kent OA et al. Transactivation of miR-34a by $\mathrm{p} 53$ broadly influences gene expression and promotes apoptosis. Mol. Cell 26(5), 745-752 (2007).
15 Lujambio A, Ropero S, Ballestar E et al. Genetic unmasking of an epigenetically silenced microRNA in human cancer cells. Cancer Res. 67(4), 1424-1429 (2007).

16 Melo SA, Ropero S, Moutinho C et al. A TARBP2 mutation in human cancer impairs microRNA processing and DICER1 function. Nat. Genet. 41(3), 365-370 (2009).

17 Saito Y, Liang G, Egger G et al. Specific activation of microRNA-127 with downregulation of the proto-oncogene BCL6 by chromatin-modifying drugs in human cancer cells. Cancer Cell 9(6), 435-443 (2006).

18 Thomson JM, Newman M, Parker JS, Morin-Kensicki EM, Wright T, Hammond SM. Extensive post-transcriptional regulation of microRNAs and its implications for cancer. Genes Dev. 20(16), 2202-2207 (2006).

19 Sevignani C, Calin GA, Nnadi SC et al. MicroRNA genes are frequently located near mouse cancer susceptibility loci. Proc. Natl Acad. Sci. USA 104(19), 8017-8022 (2007).

20 Melo SA, Esteller M. Dysregulation of microRNAs in cancer: playing with fire. FEBS Lett. 585(13), 2087-2099 (2010).

21 Calin GA, Dumitru CD, Shimizu M et al. Frequent deletions and down-regulation of micro-RNA genes miR15 and miR16 at 13 q14 in chronic lymphocytic leukemia. Proc. Natl Acad. Sci. USA 99(24), 15524-15529 (2002).

22 Costinean S, Zanesi N, Pekarsky Y et al. Pre-B cell proliferation and lymphoblastic leukemia/high-grade lymphoma in E(mu)-miR 155 transgenic mice. Proc. Natl Acad. Sci. USA 103(18), 7024-7029 (2006).

23 Klein U, Lia M, Crespo M et al. The DLEU2/miR-15a/16-1 cluster controls B cell proliferation and its deletion leads to chronic lymphocytic leukemia. Cancer Cell 17(1), 28-40 (2010).

24 Hudder A, Novak RF. miRNAs: effectors of environmental influences on gene expression and disease. Toxicol. Sci. 103(2), 228-240 (2008).

25 Iorio MV, Ferracin M, Liu CG et al. MicroRNA gene expression deregulation in human breast cancer. Cancer Res. 65(16), 7065-7070 (2005).

26 Lu J, Getz G, Miska EA et al. MicroRNA expression profiles classify human cancers. Nature 435(7043), 834-838 (2005).

27 Gaur A, Jewell DA, Liang Y et al. Characterization of microRNA expression levels and their biological correlates in human cancer cell lines. Cancer Res. 67(6), 2456-2468 (2007).

28 Yanaihara N, Caplen N, Bowman E et al. Unique microRNA molecular profiles in lung cancer diagnosis and prognosis. Cancer Cell 9(3), 189-198 (2006).

- $=$ One of the first articles to define microRNA (miRNA) expression in lung cancer patients and correlate the expression pattern with prognosis.

29 Shen YL, Jiang YG, Greenlee AR, Zhou LL, Liu LH. MicroRNA expression profiles and miR-10a target in anti-benzo[a] pyrene-7, 8-diol-9, 10-epoxide-transformed human $16 \mathrm{HBE}$ cells. Biomed. Environ. Sci. 22(1), 14-21 (2009).

30 Pogribny IP, Tryndyak VP, Boyko A, Rodriguez-Juarez R, Beland FA, Kovalchuk O. Induction of microRNAome deregulation in rat liver by long-term tamoxifen exposure. Mutat. Res. 619(1-2), 30-37 (2007).

31 Hayashita Y, Osada H, Tatematsu Y et al. A polycistronic microRNA cluster, miR-17-92, is overexpressed in human lung cancers and enhances cell proliferation. Cancer Res. 65(21), 9628-9632 (2005).

32 Ruan K, Fang X, Ouyang G. MicroRNAs: novel regulators in the hallmarks of human cancer. Cancer Lett. 285(2), 116-126 (2009).

33 Asangani IA, Rasheed SA, Nikolova DA et al. MicroRNA-21 (miR-21) posttranscriptionally downregulates tumor suppressor Pdcd4 and stimulates invasion, intravasation and metastasis in colorectal cancer. Oncogene 27(15), 2128-2136 (2008).

34 Meng F, Henson R, Wehbe-Janek H, Ghoshal K, Jacob ST, Patel T. MicroRNA-21 regulates expression of the PTEN tumor suppressor gene in human hepatocellular cancer. Gastroenterology 133(2), 647-658 (2007).

35 Vandenboom Ii TG, Li Y, Philip PA, Sarkar FH. MicroRNA and cancer: tiny molecules with major implications. Curr. Genomics 9(2), 97-109 (2008).

36 Takamizawa J, Konishi H, Yanagisawa K et al. Reduced expression of the let-7 microRNAs in human lung cancers in association with shortened postoperative survival. Cancer Res. 64(11), 3753-3756 (2004).

" $\quad$ Role of miRNA in lung cancer.

37 Wu F, Zhu S, Ding Y, Beck WT, Mo YY MicroRNA-mediated regulation of Ubc? expression in cancer cells. Clin. Cancer Res. 15(5), 1550-1557 (2009).

38 Geiss-Friedlander R, Melchior F. Concepts in sumoylation: a decade on. Nat. Rev. Mol. Cell. Biol. 8(12), 947-956 (2007).

39 Bueno MJ, Malumbres M. MicroRNAs and the cell cycle. Biochim. Biophys. Acta 1812(5), 592-601 (2011).

40 Inui M, Martello G, Piccolo S. MicroRNA control of signal transduction. Nat. Rev. Mol. Cell. Biol. 11(4), 252-263 (2010). 
41 Kong W, Yang H, He L et al. MicroRNA-155 is regulated by the transforming growth factor $\beta /$ Smad pathway and contributes to epithelial cell plasticity by targeting RhoA. Mol. Cell. Biol. 28(22), 6773-6784 (2008).

42 Xu J, Kochanek KD, Murphy SL, TejadaVera B. Deaths: Final data for 2007. Natl Vital Stat. Rep. 58, 1-135 (2010).

43 Murray CJL. The Global Burden of Disease. World Health Organization, Geneva, Switzerland; Harvard School of Public Health, Boston, MA; World Bank (1996).

44 Oglesby IK, McElvaney NG, Greene CM. MicroRNAs in inflammatory lung disease - master regulators or target practice? Respir. Res. 11, 148 (2010).

45 Sato T, Liu X, Nelson A et al. Reduced miR-146a increases prostaglandin Ein chronic obstructive pulmonary disease fibroblasts. Am. J. Respir. Crit. Care Med. 182(8), 1020-1029 (2010).

46 Pottelberge GR, Mestdagh P, Bracke KR et al. MicroRNA expression in induced sputum of smokers and patients with chronic obstructive pulmonary disease. Am. J. Respir. Crit. Care Med. 183(7), 898-906 (2011).

47 Akbas F, Coskunpinar E, Aynaci E, Musteri OY, Yildiz P. Analysis of serum micro-RNAs as potential biomarker in chronic obstructive pulmonary disease. Exp. Lung Res. 38(6), 286-294 (2012).

48 Izzotti A, Calin GA, Steele VE, Croce CM, De FS. Relationships of microRNA expression in mouse lung with age and exposure to cigarette smoke and light. FASEB J. 23(9), 3243-3250 (2009).

49 Schroen B, Heymans S. MicroRNAs and beyond: the heart reveals its treasures. Hypertension 54(6), 1189-1194 (2009).

50 Catalucci D, Gallo P, Condorelli G. MicroRNAs in cardiovascular biology and heart disease. Circ. Cardiovasc. Genet. 2(4), 402-408 (2009).

51 Sen CK, Gordillo GM, Khanna S, Roy S. Micromanaging vascular biology: tiny microRNAs play big band. J. Vasc. Res. 46(6), 527-540 (2009).

52 Haver VG, Slart RH, Zeebregts CJ, Peppelenbosch MP, Tio RA. Rupture of vulnerable atherosclerotic plaques: microRNAs conducting the orchestra? Trends Cardiovasc. Med. 20(2), 65-71 (2010).

53 Urbich C, Kuehbacher A, Dimmeler S. Role of microRNAs in vascular diseases, inflammation, and angiogenesis. Cardiovasc. Res. 79(4), 581-588 (2008).

54 Harris TA, Yamakuchi M, Ferlito M, Mendell JT, Lowenstein CJ. MicroRNA-126 regulates endothelial expression of vascular cell adhesion molecule 1. Proc. Natl Acad. Sci. USA 105(5), 1516-1521 (2008).

55 Ji R, Cheng Y, Yue J et al. MicroRNA expression signature and antisense-mediated depletion reveal an essential role of MicroRNA in vascular neointimal lesion formation. Circ. Res. 100(11), 1579-1588 (2007).

56 Martin MM, Buckenberger JA, Jiang J et al. The human angiotensin II type 1 receptor $+1166 \mathrm{~A} / \mathrm{C}$ polymorphism attenuates microrna-155 binding. J. Biol. Chem. 282(33), 24262-24269 (2007).

57 O'Connell RM, Rao DS, Chaudhuri AA et al. Sustained expression of microRNA-155 in hematopoietic stem cells causes a myeloproliferative disorder. J. Exp. Med. 205(3), 585-594 (2008).

58 Pasterkamp G, Van Keulen JK, de Kleijn DP. Role of Toll-like receptor 4 in the initiation and progression of atherosclerotic disease. Eur. J. Clin. Invest 34(5), 328-334 (2004).

59 Chen XM, Splinter PL, O'Hara SP, LaRusso NF. A cellular micro-RNA, let-7i, regulates Toll-like receptor 4 expression and contributes to cholangiocyte immune responses against Cryptosporidium parvum infection. J. Biol. Chem. 282(39), 28929-28938 (2007).

60 Taganov KD, Boldin MP, Chang KJ, Baltimore D. NF- $\kappa \mathrm{B}$-dependent induction of microRNA miR-146, an inhibitor targeted to signaling proteins of innate immune responses. Proc. Natl Acad. Sci. USA 103(33), 12481-12486 (2006).

61 Small EM, Frost RJ, Olson EN. MicroRNAs add a new dimension to cardiovascular disease. Circulation 121(8), 1022-1032 (2010).

62 Yang WJ, Yang DD, Na S, Sandusky GE, Zhang Q, Zhao G. Dicer is required for embryonic angiogenesis during mouse development. J. Biol. Chem. 280(10), 9330-9335 (2005).

63 Kuehbacher A, Urbich C, Zeiher AM, Dimmeler S. Role of Dicer and Drosha for endothelial microRNA expression and angiogenesis. Circ. Res. 101(1), 59-68 (2007).

64 Bonauer A, Carmona G, Iwasaki M et al. MicroRNA-92a controls angiogenesis and functional recovery of ischemic tissues in mice. Science 324(5935), 1710-1713 (2009).

65 Chico TJ, Milo M, Crossman DC. The genetics of cardiovascular disease: new insights from emerging approaches. J. Pathol. 220 (2), 186-197 (2010).

66 Poliseno L, Tuccoli A, Mariani L et al. MicroRNAs modulate the angiogenic properties of HUVECs. Blood 108(9), 3068-3071 (2006).
67 Tuccoli A, Poliseno L, Rainaldi G. miRNAs regulate miRNAs: coordinated transcriptional and post-transcriptional regulation. Cell Cycle 5(21), 2473-2476 (2006).

68 Suarez Y, Fernandez-Hernando C, Pober JS, Sessa WC. Dicer dependent microRNAs regulate gene expression and functions in human endothelial cells. Circ. Res. 100(8), 1164-1173 (2007).

69 Cordes KR, Srivastava D. MicroRNA regulation of cardiovascular development. Circ. Res. 104(6), 724-732 (2009).

70 Fasanaro P, Greco S, Lorenzi M et al. An integrated approach for experimental target identification of hypoxia-induced miR-210. J. Biol. Chem. 284(50), 35134-35143 (2009).

71 Simonneau G, Robbins IM, Beghetti M et al. Updated clinical classification of pulmonary hypertension. J. Am. Coll. Cardiol. 54(Suppl. 1), S43-S54 (2009).

72 McGoon M, Gutterman D, Steen V et al. Screening, early detection, and diagnosis of pulmonary arterial hypertension: ACCP evidence-based clinical practice guidelines. Chest 126(Suppl. 1), S14-S34 (2004).

73 Caruso P, MacLean MR, Khanin R et al. Dynamic changes in lung microRNA profiles during the development of pulmonary hypertension due to chronic hypoxia and monocrotaline. Arterioscler. Thromb. Vasc. Biol. 30(4), 716-723 (2010).

74 Courboulin A, Paulin R, Giguere NJ et al. Role for miR-204 in human pulmonary arterial hypertension. J. Exp. Med. 208(3), 535-548 (2011).

75 Tsitsiou E, Lindsay MA. MicroRNAs and the immune response. Curr. Opin. Pharmacol. 9(4), 514-520 (2009).

76 Sonkoly E, Stahle M, Pivarcsi A. MicroRNAs and immunity: novel players in the regulation of normal immune function and inflammation. Semin. Cancer Biol. 18(2), 131-140 (2008).

77 Perry MM, Moschos SA, Williams AE, Shepherd NJ, Larner-Svensson HM, Lindsay MA. Rapid changes in microRNA-146a expression negatively regulate the IL-1betainduced inflammatory response in human lung alveolar epithelial cells. J. Immunol. 180 (8), 5689-5698 (2008).

78 Izzotti A, Calin GA, Arrigo P, Steele VE, Croce CM, De Flora S. Downregulation of microRNA expression in the lungs of rats exposed to cigarette smoke. FASEB J. 23(3), 806-812 (2009).

" First study to examine cigaratte smokeinduced changes in miRNA expression in the lung.

79 Spira A, Beane J, Shah V et al. Effects of cigarette smoke on the human airway 
epithelial cell transcriptome. Proc. Natl Acad. Sci. USA 101(27), 10143-10148 (2004).

80 Kalscheuer S, Zhang X, Zeng Y, Upadhyaya P. Differential expression of microRNAs in early-stage neoplastic transformation in the lungs of F344 rats chronically treated with the tobacco carcinogen 4-(methylnitrosamino)-1-(3-pyridyl)-1butanone. Carcinogenesis 29(12), 2394-2399 (2008).

81 Izzotti A, Larghero P, Cartiglia C et al. Modulation of microRNA expression by budesonide, phenethyl isothiocyanate and cigarette smoke in mouse liver and lung. Carcinogenesis 31(5), 894-901 (2010).

" First study to show dysregulation of miRNA expression in lung and liver by environmental cigaratte smoke.

82 Izzotti A, Larghero P, Balansky R, Pfeffer U, Steele VE, De FS. Interplay between histopathological alterations, cigarette smoke and chemopreventive agents in defining microRNA profiles in mouse lung. Mutat. Res. 717(1-2), 17-24 (2011).

83 Izzotti A, Larghero P, Longobardi $\mathrm{M}$ et al. Dose-responsiveness and persistence of microRNA expression alterations induced by cigarette smoke in mouse lung. Mutat. Res. 717(1-2), 9-16 (2011).

84 Russ R, Slack FJ. Cigarette-smoke-induced dysregulation of microRNA expression and its role in lung carcinogenesis. Pulm. Med. 2012, 791234 (2012).

85 Schembri F, Sridhar S, Perdomo C et al. MicroRNAs as modulators of smokinginduced gene expression changes in human airway epithelium. Proc. Natl Acad. Sci. USA 106(7), 2319-2324 (2009).

w- Reports cigarette smoke-induced dysregulation of miRNA in humans.

86 Christenson SA, Schembri FM, Sridhar S et al. Impact of smoking on miRNA expression in buccal and nasal epithelium. Am. J. Respir. Crit. Care Med. (179), 457-466 (2009).

87 Swan GE, Hodgkin JE, Roby T, Mittman C, Jacobo N, Peters J. Reversibility of airways injury over a 12 -month period following smoking cessation. Chest 101(3), 607-612 (1992).

88 Moriyama C, Betsuyaku T, Nishimura M. Effects of aging and smoking on MicroRNA expression profiling in bronchiolar epithelium. Am. J. Respir. Crit. Care Med. 181, A2025 (2010).

89 Perdomo C, Spira A, Schembri F. MiRNAs as regulators of the response to inhaled environmental toxins and airway carcinogenesis. Mutat. Res. 717(1-2), 32-37 (2011).

90 Jardim MJ, Fry RC, Jaspers I, Dailey L, Diaz-Sanchez D. Disruption of microRNA expression in human airway cells by diesel exhaust particles is linked to tumorigenesisassociated pathways. Environ. Health Perspect. 117(11), 1745-1751 (2009).

91 Biomarkers Definition Working Group. Biomarkers and surrogate endpoints: preferred definitions and conceptual framework. Clin. Pharmacol. Ther. 69(3), 89-95 (2001).

92 Waldman SA, Terzic A. Translating MicroRNA discovery into clinical biomarkers in cancer. JAMA 297(17), 1923-1925 (2007).
93 Mascaux C, Laes JF, Anthoine G et al. Evolution of microRNA expression during human bronchial squamous carcinogenesis. Eur. Respir. J. 33(2), 352-359 (2009).

- - First study to show involvement of miRNA from the earliest steps of bronchial carcinogenesis.

94 Rabinowits G, Gercel-Taylor C, Day JM, Taylor DD, Kloecker GH. Exosomal microRNA: a diagnostic marker for lung cancer. Clin. Lung Cancer 10(1), 42-46 (2009).

- One of the studies suggesting that miRNA in blood could possibly be a predictive biomarker of disease.

95 Weber JA, Baxter DH, Zhang S et al. The microRNA spectrum in 12 body fluids. Clin. Chem. 56(11), 1733-1741 (2010).

- Concentrations of specific miRNAs in body fluids are potential biomarkers for the diagnosis and monitoring of various physiopathological conditions.

96 Small EM, Olson EN. Pervasive roles of microRNAs in cardiovascular biology. Nature 469(7330), 336-342 (2011).

97 Fish JE, Santoro MM, Morton SU et al. miR-126 regulates angiogenic signaling and vascular integrity. Dev. Cell 15(2), 272-284 (2008).

\section{Website}

101 MiRBase.

www.mirbase.org/cgi-bin/browse.pl 